\title{
Family and Mental Illness
}

\author{
M.Senthil ${ }^{1}$, Swapnil Vidyarthi ${ }^{2}$, Dr. Manisha Kiran ${ }^{3}$ \\ 1. Assistant Professor of Social Work, Dept of Social Work, Central University of Tamilnadu, Tamilnadu, \\ Thiruvarur, Tamilnadu, India. \\ 2. Mphil in Psychiatric Social Work, Dept of Psychiatric Social Work, Central Institute of Psychiatry, Ranchi, \\ Jharkhand, India. \\ 3. Assoc. Prof \& Head., Dept of Psychiatric Social Work, Ranchi Institute Neuro Psychiatry \& Allied Sciences \\ (RINPAS), Ranchi, Jharkhand,India.
}

\begin{abstract}
A family is an exclusive group of people who share a close relationship - a unit typically composed of a matedcouple and their dependent children in co-residence. Families create generations-each of which gain in maturity and self-sufficiency such as to create and provide for subsequent generations. The word 'family' came from the Latin word familiare. Family is a group of people who have tied by kinship. Family has been the society's primaryagency in satisfying common needs for survival, sense of loving and belonging, status andself-esteem, and self-realization since the very onset of human civilization. The family provides for the child's biological needs andsimultaneously directs its development towards becoming an integrated person capableof living in society and maintaining and transmitting culture. Specific to mental health, family plays a very significant role in development of positive mental health and making a person psychologically resourceful and socially organizedFamily factors in conjunction with biological and genetic factors could play instrumental role in causing psychological problems. Chances of occurring of psychological problems could be lessened if family climate is maintained as healthy and supportive to family members-soothing family atmosphere could work as a buffering agent against stress and limit the deleterious effect of stress on one's psyche.
\end{abstract}

Keywords: Dysfunctional Family, Family, and Mental Illness.

\section{Introduction}

A family is an exclusive group of people who share a close relationship - a unit typically composed of a matedcouple and their dependent children in co-residence. Families create generations - each of which gain in maturity and self-sufficiency such as to create and provide for subsequent generations. The word 'family' came from the Latin word familiare. Family is a group of people who have tied by kinship. Family has been the society's primaryagency in satisfying common needs for survival, sense of loving and belonging, status andselfesteem, and self-realization since the very onset of human civilization. The family provides for the child's biological needs andsimultaneously directs its development towards becoming an integrated person capableof living in society and maintaining and transmitting culture. Specific to mental health, family plays a very significant role in development of positive mental health and making a person psychologically resourceful and socially organized (David, 1978). The past few decades especially after 1950s researchers have seen showing clinical and scientific interest over family and its role as one of the most significant social forces in human development (David, 1978). Sociologists aim to delineate institutional characteristics of the family and the health effects of socioeconomic status. Anthropologists aim to discuss about family structure keeping in view of diverse cultures and societies or role of society and culture in day-to-day as well as longitudinal family processes and operations. Researchers of Systemic School of Psychology and Family Study put their attention to explain the role of the whole family in causing psychological disturbance to individual member, the impact of disorder on family life, and the way the family contributes to illness and health care (David, 1978). Family and other primary environmental components have key role in developing interpersonal relationship skills and abilities of people. Inadequate and faulty interpersonal skill might become the pivotal factor for shaping up one's personality as problematic one. Presently biological hypotheses of different psychiatric disorders take over the field of psychiatry and mental health but the role of environment in causing psychiatric disorders is no less important. It isimportant to emphasize that relationships with important otherslike core family members may play a crucial role in individual outcome. Psychoanalytictheories in the form of object relations, self psychology, andrelational psychoanalysis reflect this emphasis, and at a broaderlevel, the interpersonal school of psychiatry focuses selectivelyon the role of relationships in health and illness (Lewis, 1998). 


\section{Types of Family}

Most of the time when a person thinks of the definition of a family, the image of a mother, father and children is what comes into the mind. That is actually the definition of a nuclear family, which is parents, and one or more children. However, there are more definitions that can be used to define a family such as a singleparent family which is one parent and a child or children. Extended family is when a nuclear family or singleparent family lives with any extended family members

- Nuclear Family/Conjugal Family = husband, the wife, and unmarried children.

- Single Parent Family = family consists of one parent and children

- Extended Family = many generations living under the same roof, depending on the circumstances.

- Childless Couples

- Matrilocal Family = consists of a mother and her children. Commonly exists where women have to rear their children by themselves, or where men are more mobile than women.

- Joint Family (Indianized form) $=$ in India join family concept is still prevailing. It consists of all persons lineally descended from a common ancestor, and includes their wives and children. A daughter ceases to be a member of her father's family on marriage and becomes a member of her husband's family. In joint family parents and their children's (usuallly males) families live under a single roof. This type of family includes multiple generations in the family. Jointness and collaborativeness are found in family's economic, financial, property related, daily functioning, cultural-religious, and social activities.

\section{Areas of family functioning:}

Healthy families generally promote as well as protect the emotional, physical and social welfare of individual family members. Among the many factors that contribute to this process are a family's internal strengths and the durability of the family unit. Unlike any other social group, families are able to provide the close emotional support needed to produce self-confident and well-adjusted children and adults. Likewise, families that function in a healthy manner are well equipped to deal with the many normal changes and unexpected crises that confront them throughout their lifetime. Therefore, the family's primary function is to create a healthy environment where family members can successfully grow and develop. To be emerged as a healthy family unit a given family unit must have optimal performances in following areas:

\section{a) Family task areas:}

The family's task areas include basic, developmental and crisis tasks. The basic task area is concerned with the provision and appropriate distribution of food, money, shelter and other necessities of life among members. The developmental tasks include individual and family stages of growth. Individual developmental stages include infancy, childhood, adolescence, adulthood, and aging. The family developmental tasks are stages in the family life cycle. These stages include the marriage and early years before children; the childbearing family; the family with school age children; the family with teenagers; the family launching center; the family/middle years; and the aging family. Crisis tasks are family hardship events such as illness, job loss, accidents, relocation, or death. Families that are able to cope with and adapt to stressful life events and transitions are better able to maintain a healthy family environment. Healthy families are able to cope with and adapt to stressful life events and transitions.

\section{b) Problem-solving}

Problem-solving is defined as a family's ability to resolve problems on a level that maintains effective family functioning (Epstein et al., 1993). A problem is an issue without an easy solution that will threaten the family's ability to function if it is not resolved. Problem-solving is the family's ability to resolve problems on a level that maintains effective family functioning (Epstein et al., 1993). Family problems come in all shapes and sizes. Some problems involve everyday decisions about money or transporting the children to and from school. These are called instrumental problems. Other problems may be concerned with a family member's feelings and emotions and are called affective issues.

\section{c) Communication}

Communication is defined as the way verbal and nonverbal information is exchanged within a family (Epstein et al., 1993). Effective family communication depends on several factors, including clear and direct communication between family members. Families who can express their feelings to one another are better equipped to solve problems as they arise. The ability to listen to others and to pay attention to what they say are essential skills for effective family communication. 


\section{d)Family roles}

Family roles are recurrent patterns of behavior by which family members fulfill family functions (Epstein et al., 1993). The establishment of clear roles within a family is directly connected to a family's ability to deal with normal and unexpected changes. Healthy families are able to establish clear, yet flexible, roles that enable them to carry out family functions. Deciding work roles inside and outside the home is an important family task.

\section{e)Affective responsiveness}

Affective responsiveness is the family's ability to respond emotionally to other family members in an appropriate manner (Epstein et al., 1993). Families need to be able to share and experience feelings such as love, tenderness, joy, fear, and anger. Families that are unable to respond, for example, with sadness or tenderness, may be restricted or even distorted emotionally.

\section{f) Affective involvement}

Affective involvement is how well the family as a whole shows interest in and values the activities and interests of individual family members (Epstein et al., 1993). Both overinvolvement and under-involvement are patterns of behavior that can pose problems for families.

\section{g)Behavior control}

Behavior control refers to patterns of behavior that the family adopts for dealing with family situations (Epstein et al., 1993). Some families have flexible behavior patterns while others may have more rigid patterns. Families with flexible behavior patterns are better able to adjust to and cope with changing family circumstances.

\section{h)Family Strengths}

Families can make a difference in the lives of their children. Research has identified several factors that promote resiliency in children. Among these are social competence, problem-solving skills, autonomy and a sense of purpose and future (National Network for Family Resiliency, 1993). Children whose families promote these skills have a better chance of becoming successful adults. In addition, families who express caring and support, create high expectations for family members, and encourage children's participation in school and other activities are more likely to have happy and successful family members.

\section{Marital problems and psychological disorder}

Marital disharmony could play an important role in causing psychological problems to affected individuals. Marital relationship is a major source of support to people; if it gets into turmoil then both the persons miss it. In a large scale population study on females Kendler et al (1995) found that family related factors like death of a close relative, assault, serious marital problems, divorce and separation can evoke major depression to those people who have genetic predisposition. One hypothesis, i.e., the Marital Discord Model of Depression theorizes that marital discord is an important risk factor for depression among many married people (Beach et al., 1990). Although authors of this model recognize that the aetiology of depression is often multifaceted, still they state that factors like decreased social support and increased hostility in troubled relationships can precipitate depressive symptomotology. In case of schizophrenia often marital problem occurs and results into breaking off relationship between patient and his/her spouse. It was seen earlier schizophrenia patients who remained for long periods in hospital were more often divorced or separated than those whose illness was less chronic (Dominian, 1979). In case of anxiety and neurotic disorders, Ryle (1967) found that neuroticism was associated with marital breakdown and poor marital adjustment, as measured by the gap between affection given and received. Kreitman (1970) found that male neurotics tend to be less affectionate to their wives' than normal persons. A neurotic husband tends to lack reliability and be emotionally irresponsible, impulsive, and antisocial. He is also likely to sulk, make scenes, and be aggressive. Such husbands also resist their wives' independence, which thus restricts their leisure activities. The inability of the wife to escape from her husband's constant neurotic behaviour may contribute to her marital tension. Marital tension is, in fact, associated with behaviour in the spouse that is either unilaterally dominating or segregating.

\section{Domestic violence, abuse and psychological disorder}

Domestic violence or spousal violence has been emerging as a pervasive global issue, contributes significantly to preventable morbidity and mortality for people across diverse cultures. In major cases women have been found to be at receiving end of violence. There are ample evidences which suggest that domestic violence can have profound physical and psychological sequelae (Fischbach\& Herbert, 1997). Women who witness regular domestic violence from their partners or spouses have the risks of developing depression and anxiety disorders (Humphreys \&Thiara, 2003). 


\section{What is a dysfunctional family?}

A dysfunctional family is a family, in which conflict, misbehavior and even abuse on the part of individual members of the family occur continually, leading other members to accommodate such actions. Children sometimes grow up in such families with the understanding that such an arrangement is normal. Dysfunctional families are most often a result of the alcoholism, substance abuse, or other addictions of parents, parents' untreated mental illnesses/defects or personality disorders, or the parents emulating their own dysfunctional parents and dysfunctional family experiences. Violence and verbal abuse are typical outcomes. Choosing one or more of an appropriate twelve-step program has been found to be of great help to all the family involved. Dysfunctional family members have common symptoms and behavior patterns as a result of their common experiences within the family structure. This tends to reinforce the dysfunctional behavior, either through enabling or perpetuation. The family unit can be affected by a variety of factors.

Family dysfunction can be any condition that interferes with healthy family functioning. Most families have some periods of time where functioning is impaired by stressful circumstances (death in the family, a parent's serious illness, etc.). Healthy families tend to return to normal functioning after the crisis passes. In dysfunctional families, however, problems tend to be chronic and children do not consistently get their needs met. Negative patterns of parental behavior tend to be dominant in their children's lives.

Sexual abuse happens to both boys and girls. It is perpetrated by both men and women. It cuts across lines of race, socioeconomic level, education level, and religious affiliation. In most cases, sexual abuse is part of an overall family pattern of dysfunction, disorganization, and inappropriate role boundaries. Responsibility for sexual abuse in all cases rests entirely with the adult. No child is responsible for being abused. Most sexually abused children are too frightened of the consequences for themselves and their families to risk telling another adult what is happening. As a result they grow into adulthood carrying feelings of self-loathing, shame, and worthlessness. They tend to be self-punishing and have considerable difficulties with relationships and with sexuality. Regardless of the kind of dysfunction or abuse, effects vary widely across individuals. Support from other healthy adults, success in other areas, or positive changes in the family can help prevent or minimize negative effects. The following questions may help you identify how you may have been or continue to be affected (Beavers, 1982; Ackerman, 1993).

\section{Types of dysfunctional families}

According to Janet Kizziar identified four types of "troubled or dysfunctional family systems," in the context of describing the cardinal features of alcoholic families, such as:

- The Alcoholic or Chemically Dependent Family System

- The Emotionally or Psychologically Disturbed Family System

- The Physically or Sexually Abusing Family System

- The Religious Fundamentalist or Rigidly Dogmatic Family System

Kizziar (1989) mentioned that in all those families substance addiction or alcoholism tends to become more problematic as other non-dependent members of the family somehow promote or fuel the evil habit by becoming reluctant to them, or quietly accepting their addictive behaviour. Steven Farmer (1989) identified following symptoms in dysfunctional families:

- Inconsistency and Unpredictability

- Role reversals ("parentifying" children)

- Closed family system" (a socially isolated family that discourages relationships with outsiders)

- Denial (i.e. a refusal to acknowledge the alcoholism of a family member; ignoring complaints of physical, emotional and sexual abuse)

- Lack of empathy toward family members

- Lack of clear boundaries (i.e. throwing away personal possessions that belong to others, inappropriate touching, etc.)

- $\quad$ Mixed Messages

- Extremes in conflict (either too much or too little fighting between family members)

Later Neurath (2002) also categorized dysfunctional family by identifying faulty parenting as:

- Dogmatic or chaotic parenting" (applying harsh and inflexible disciplines on children)

- Showing condition-based love and affection to children

- Socially isolated parents or parents with low social mixing skills

- Children are not expected to question parents or children not allowed to dissent or argument with parents

- Children are not allowed to develop their own value system by parents

- Parents are disrespectful to children's need, prestige and existence 
- Signs of emotional intolerance from parents i.e., family members not allowed to express the "wrong" emotions

\section{a) Deficient Parents:}

\section{Examples of Faulty Parenting in Dysfunctional Families}

Deficient parenting is characterized by having marked inadequacies in parents to provide children emotional and material care. These parents would likely to hurt their children more by omission than by commission. Frequently, chronic mental illness or a disabling physical illness contributes to parental inadequacy. Children tend to take on adult responsibilities from a young age in these families. Parental emotional needs tend to take precedence, and children are often asked to be their parents' caretakers. Children are robbed of their own childhood, and they learn to ignore their own needs and feelings. Because these children are simply unable to play an adult role and take care of their parents, they often feel inadequate and guilty. These feelings continue into adulthood.

\section{b) Controlling Parents:}

Unlike the deficient parents, controlling parents fail to allow their children to assume responsibilities appropriate for their age. These parents generally show excessive dominating attitude to children and making decisions for their children well beyond the age at which this is necessary. Controlling parents are often driven by a fear of becoming unnecessary to their children. This fear leaves them feeling betrayed and abandoned when their children become independent. On the other hand, these children frequently feel resentful, inadequate, and powerless. Transitions into adult roles are quite difficult, as these adults frequently have difficulties making decisions independent from their parents. When they act independently these adults feel very guilty, as if growing up were a serious act of disloyalty.

\section{c) Alcoholic Parents:}

Alcoholic families tend to be chaotic and unpredictable. Rules those are applicable to one day may not be applicable in other days. Promises are neither kept nor remembered. Expectations vary from one day to the next. Parents may be strict at times and indifferent at others. In addition, emotional expression is frequently forbidden and discussion about the alcohol use or related family problems is usually nonexistent. Family members are usually expected to keep problems a secret, thus preventing anyone from seeking help. All of these factors leave children feeling insecure, frustrated, and angry. Children often feel there must be something wrong with them which make their parents behave this way. Mistrust of others, difficulty with emotional expression, and difficulties with intimate relationships carry over into adulthood. Children of alcoholics are at much higher risk for developing alcoholism than are children of non-alcoholics.

\section{d) Abusive Parents:}

Abuse can be verbal, physical, or sexual. Verbal abuse - such as frequent criticism, being critical to children's behaviour, frequently putting down children in front of others, comparing with other children regarding their academic, scholastic, behavioural, even physical appearances and showing less positive reinforcements - can have enduring effects, particularly when it comes from those entrusted with the child's care. Some verbal abusers are very direct, while others use subtle put-downs disguised as humor. Both types are equally damaging to children. Definitions of physical abuse vary widely. Many parents, at one time or another, have felt the urge to strike their child. With physically abusive parents, however, the urge is frequent and little effort is made to control this impulse. The Federal Child Abuse Prevention and Treatment Act (U.S. Department of Health and Human Services, Administration for Children and Families) defines physical abuse as "the infliction of physical injuries such as bruises, burns, welts, cuts, bone or skull fractures; these are caused by kicking, punching, biting, beating, knifing, strapping, paddling, etc." Physically abusive parents can create an environment of terror for the child, particularly since violence is often random and unpredictable. Abused children often feel anger. Children of abusive parents have tremendous difficulties developing feelings of trust and safety even in their adult lives. While parents may justify or rationalize verbal or physical abuse as discipline aimed at somehow helping the child, there is no rationalization for sexual abuse. Sexual abuse is the most blatant example of an adult abusing a child purely for that adult's own gratification. Sexual abuse can be any physical contact between an adult and child where that contact must be kept secret. Demonstrations of affection -- such as hugging, kissing, or stroking a child's hair -- that can be done openly are quite acceptable and even beneficial. When physical contact is shrouded in secrecy then it is most likely inappropriate. Sexual abuse happens to both boys and girls. It is perpetrated by both men and women. It cuts across lines of race, socioeconomic level, education level, and religious affiliation. In most cases, sexual abuse is part of an overall family pattern of dysfunction, disorganization, and inappropriate role boundaries. Responsibility for sexual abuse in all cases rests entirely with the adult. No child is responsible for being abused. Most sexually abused 
children are too frightened of the consequences for themselves and their families to risk telling another adult what is happening. As a result they grow into adulthood carrying feelings of self-loathing, shame, and worthlessness. They tend to be self-punishing and have considerable difficulties with relationships and with sexuality.

\section{Effects of faulty parenting on children}

not healthy:

Children growing up in a dysfunctional family may have to adopt following six basic roles, which are

a) The Good Child - a child who takes over the parental role.

b) The Problem Child - the child who has been held responsible for most problems in his family, although he is found to be the most emotionally stable one in the family.

c) The Caretaker Child- the child who takes the maximum responsibility for the emotional well-being of the family.

d) The Lost Child - the inconspicuous, quiet one, whose needs are often ignored or hidden by family members.

e) The Mascot Child- the child who frequently uses humours or adopts the role of a comedian to divert attention away from the increasingly dysfunctional family system.

f) The Mastermind Child- the child who has opportunistic attitudes and who capitalizes on the other family members' faults in order to get whatever he/she wants.

\section{Conclusion}

Family factors in conjunction with biological and genetic factors could play instrumental role in causing psychological problems. Chances of occurring of psychological problems could be lessened if family climate is maintained as healthy and supportive to family members- soothing family atmosphere could work as a buffering agent against stress and limit the deleterious effect of stress on one's psyche.

[1]. Ackerman, R.J. (1993). Silent Sons. Simon \& Schuster.

[2]. Beach, S.R.H., Sandeen, E.E., O'Leary, K.D. (1990). Depression in marriage. New York: Guildford Press. As cited in: C.S. Hollist, R. Miller, O.G. Falceto, C.L.C. Fernandes. (2007). Marital satisfaction and depression: A replication of the marital discord model in a Latino sample. Family Process 46 (4), 485-498. Downloaded from http://digitalcommons.unl.edu/famconfacpub/48.

[3]. Beavers, W.R. (1982). Healthy, midrange and severely dysfunctional families. In F. Walsh (Ed.), Normal Family Process. New York: Guilford Press, pp.45-66. Excerpts taken from Books Google on 04/07/2010.

[4]. David, H.P. (1978). Healthy family functioning: A cross-cultural appraisal. Bulletin of the World Health Organization, 56(3), 327342.

[5]. Dominian, J. (1979). Marriage and psychiatric illness. British Medical Journal, 2, 854-855.

[6]. Epstein, N., Bishop, D.S., Levin, S. (1978). The McMaster model of family functioning. Journal of Marriage and Family Counselling, 4, 19-31. (Abstract)

[7]. Fischbach, R.L. \& Herbert, B. (1997). Domestic violence and mental health: Correlates and conundrums within and across cultures. Social Science and Medicine, 45(8), 1161-1176. (Abstract)

[8]. Humphreys, C. \&Thiara, R. (2003). Mental health and domestic violence: 'I call it symptom of abuse'. British Journal of Social Work, 33, 209-226. (Abstract)

[9]. Kendler, K.S., Kessler, R.C., Walters, E.E., MacLean, C., Neale, M.C., Heath, A.C., Eaves, L.J. (1995). Stressful life events, genetic liability, and onset of an episode of major depression in women. American Journal of Psychiatry, 152, 833-842.

[10]. Kizziar, J. (1989). Excepts taken from the website: http://www.mudrashram.com/dysfunctionalfamily on 10/07/2010.

[11]. Kreitman, N, et al, British Journal of Psychiatry, 1970, 117, 33. As cited in: J. Dominian (1979). Marriage and psychiatric illness. British Medical Journal, 2, 854-855.

[12]. Lewis, J.M. (1998). For better or worse: Interpersonal relationships and individual outcome. American Journal of Psychiatry,155, 582-589.

[13]. National Network for Family Resiliency (1993). Taken from http://www.nnfr.org/

[14]. Ryle, A. (1967). Neurosis in the Ordinary Family. London, Tavistock Publications. As cited in: J. Dominian (1979). Marriage and psychiatric illness. British Medical Journal, 2, 854-855.

[15]. Thara, R., Padmavati, R., Kumar, S., Srinivasan, L. (1998) Burden Assessment Schedule: Instrument to assess burden on caregivers of chronically mentally ill. Indian Journal of Psychiatry, 40, 21-29.

[16]. U.S. Department of Health and Human Services, Administration for Children and Families. Available at http://www.acf.hhs.gov/programs/cb/laws_policies/cblaws/capta/. Taken on 14/07/2010. 